\title{
Fifty-six percent of proximal femoral cortical hypertrophies 6 to 10 years after Total hip arthroplasty with a short Cementless curved hip stem - a cause for concern?
}

\author{
Moritz M. Innmann', Johannes Weishorn', Thomas Bruckner², Marcus R. Streit ', Tilman Walker',
} Tobias Gotterbarm ${ }^{3}$, Christian Merle ${ }^{1}$ and Michael W. Maier ${ }^{1 *}$

\begin{abstract}
Background: Thigh pain and cortical hypertrophies $(\mathrm{CH})$ have been reported in the short term for specific short hip stem designs. The purpose of the study was to investigate 1) the differences in clinical outcome, thigh pain and stem survival for patients with and without $\mathrm{CHs}$ and 2) to identify patient and surgery-related factors being associated with the development of $\mathrm{CHs}$.

Methods: A consecutive series of 233 patients with 246 hips was included in the present retrospective diagnostic cohort study, who had received a total hip arthroplasty (THA) between December 2007 and 2009 with a cementless, curved, short hip stem (Fitmore, Zimmer, Warsaw, IN, USA). Clinical and radiographic follow-up, including the radiographic parameters for hip geometry reconstruction, were prospectively assessed 1, 3, and 6 to 10 years after surgery.
\end{abstract}

Results: Cortical hypertrophies were observed in 56\% of the hips after a mean of 7.7 years, compared to 53\% after 3.3 years being mostly located in Gruen zone 3 and 5. There was no significant difference for the Harris Hip Score and UCLA score for patients with and without CHs. Only one patient with a mild $\mathrm{CH}$ in Gruen zone 5 and extensive heterotopic ossifications around the neck of the stem reported thigh pain. The Kaplan Meier survival rate after 8.6 years was 99.6\% (95\%-Cl; 97.1-99.9\%) for stem revision due to aseptic loosening and no association with CHs could be detected. Postoperative increase in hip offset was the only risk factor being associated with the development of $\mathrm{CH}$ s in the regression model $(\Delta \mathrm{HO} ; \mathrm{OR} 1.1(1.0-1.2) ; p=0.001)$.

Conclusions: The percentage of cortical hypertrophies remained almost constant in the mid-term compared to the short-term with the present cementless short hip stem design. The high percentage of cortical hypertrophies seems not be a cause for concern with this specific implant in the mid-term.

Level of evidence: Diagnostic Level IV

Keywords: Cortical, Hypertrophy, Thigh, Pain, Short, Stem, Cementless, Hip, Arthroplasty

\footnotetext{
* Correspondence: Michael.Maier@med.uni-heidelberg.de

${ }^{1}$ Department of Orthopaedics and Trauma Surgery, Heidelberg University

Hospital, Schlierbacher Landstrasse 200a, 69118 Heidelberg, Germany

Full list of author information is available at the end of the article
}

(c) The Author(s). 2019 Open Access This article is distributed under the terms of the Creative Commons Attribution 4.0 International License (http://creativecommons.org/licenses/by/4.0/) which permits unrestricted use, distribution, and reproduction in any medium, provided you give appropriate credit to the original author(s) and the source, provide a link to the Creative Commons license, and indicate if changes were made. The Creative Commons Public Domain Dedication waiver (http://creativecommons.org/publicdomain/zero/1.0/) applies to the data made available in this article, unless otherwise stated. 


\section{Background}

Short hip stems show excellent survival rates ranging from 95 to $100 \%$ after 3 to 11 years, comparable to conventional cementless stems [1-6], but there is a lack of long-term data. Some disadvantages have been reported with short stems. Thigh pain occurred in $25 \%$ of patients after 2 to 4 years with the Tri-Lock Bone Preservation Stem (DePuy,Warsaw, IN,USA) and correlated with younger patient age, while only $2 \%$ of the hips showed cortical hypertrophy $(\mathrm{CH})$ [7]. In contrast, a high rate of 29 to $63 \%$ for proximal femoral $\mathrm{CHs}$ was reported after 1.3 to 3.3 years with a cementless, curved, short stem (Fitmore, Zimmer, Warsaw, IN, USA), which is subject to the present investigation, while only $4 \%$ of the patients reported thigh pain $[5,8]$.

There is little evidence in the literature on factors contributing to the development of thigh pain and cortical hypertrophies and how they are associated [5, 7]. It has been hypothesized, both clinical findings might be related to the modulus mismatch between the distal part of the stem and the proximal femur affecting bone remodeling [7, 9]. This hypothesis is supported by a biomechanical study examining the mediolateral bending behavior of cementless stems, demonstrating a higher rigidity for a short compared standard tapered stem [9]. However, the definite relationship between thigh pain, cortical hypertrophies, load transmission and proximal implant loosening is still a matter of debate. As thigh pain and cortical hypertrophies may be of high clinical relevance with short hip stems, mid-term studies with adequate study cohort size are needed.

Therefore the present retrospective cohort study questioned

1) What are the differences in clinical outcome, thigh pain and stem survival for patients with and without $\mathrm{CHs}$ and

2) What patient and surgery-related factors are associated with the development of $\mathrm{CHs}$ ?

\section{Methods}

\section{Study cohort}

The present retrospective diagnostic cohort study investigated a consecutive series of 233 patients with 246 cementless THAs from a single academic institution. The short-term results on the first 100 THAs have been previously published by the senior author in BMC Musculoskeletal Disorders reporting a percentage of $63 \%$ for proximal femoral $\mathrm{CHs}$ after 3.3 years [5]. In order to achieve a sufficient statistical power, all 233 patients were included in the study group, who had received a cementless THA between December 2007 and 2009 with a specific bone preserving curved stem (Fitmore stem, Zimmer, Warsaw, IN, USA), including the patients who had been followed in the previous short term study [5]. Indications for receiving the short stem were absence of severe proximal femoral deformity, adequate bone stock for cementless fixation and the diagnosis of primary osteoarthritis, developmental dysplasia of the hip (Crowe grade I), avascular necrosis of the femoral head, posttraumatic osteoarthritis, rheumatoid arthritis, slipped epiphysis of the femoral head or Perthes disease. The stem design for implantation was selected independently by each surgeon based on best endosteal press-fit fixation in the proximal femur and most accurate hip geometry reconstruction for hip offset and leg length difference according to templating [10]. Patients with bilateral THA or prior hip surgery were included in the study cohort. Diagnoses leading to THA and patient demographics are given in Table 1. In deceased patients, revision surgery between the last clinical follow-up and death was excluded using information from relatives, health insurance, general practitioners and clinical notes. Informed consent was obtained by all patients. The study was approved by the institutional review board (S-083/2017) and conducted according to the Helsinki Declaration of 2008.

\section{Implants \& Surgery}

A cementless, curved, short stem was used in all patients (Fitmore stem, Zimmer, Warsaw, IN, USA). The titanium alloy stem (Ti Al6V4) has a porolock Ti-VPS coating in the proximal part to enhance bone ingrowth and is available in four different neck angle options $\left(127^{\circ}\right.$, $\left.129^{\circ}, 137^{\circ}, 140^{\circ}\right)[8,11]$. The stem has a triple-tapered design to achieve press-fit fixation at the metaphyseal/ diaphyseal level and according to the recommended femoral neck resection level, this stem can be classified as a trochanter-sparing or neck-harming short stem (Fig. 1) $[5,12,13]$. A cementless press-fit cup was used in 243 THAs (Allofit cup, Zimmer, Warsaw, IN, USA, 241 hips; Pinnacle cup, DePuy Synthes, West Chester, PA, USA, 2 hips). A reinforcement ring was used in two THAs due to one dysplastic and one posttraumatic acetabulum (Ganz reinforcement ring, Zimmer, Warsaw, IN, USA). A cemented cup was used in one THA due to osteopenia of the acetabular bone (Durasul, Zimmer, Warsaw, IN, USA).

Ceramic heads with three neck length options $(-4,0$, $4 \mathrm{~mm}$; $28 \mathrm{~mm}$ or $32 \mathrm{~mm}$ diameter; Biolox forte ${ }^{\oplus}$, CeramTec, Plochingen, Germany; 234 hips) or a CoCr femoral head with one neck length option $(8 \mathrm{~mm} ; 32 \mathrm{~mm}$ diameter; Metasul, Zimmer, Warsaw, IN, USA, 11 hips; Articul, DePuy Synthes, West Chester, PA, USA, one hip)) articulated with a highly cross-linked polyethylene liner (Durasul ${ }^{\ominus}$; Zimmer, Warsaw, IN, USA). Hard on soft bearings with highly cross linked polyethylene liners were used in 244 THAs and ceramic on ceramic 
Table 1 Demographics and diagnosis for patients with and without cortical hypertrophies

\begin{tabular}{|c|c|c|c|}
\hline & Hips with $\mathrm{CHs}$ & Hips without $\mathrm{CHs}$ & $p$-value \\
\hline \multicolumn{4}{|l|}{ Demographics } \\
\hline Number of hips & 105 & 83 & \\
\hline Gender (m: w) & 57: 48 & 29: 44 & 0.32 \\
\hline Age at surgery in years & $61(53-68)$ & $60(51-68)$ & 0.92 \\
\hline BMI $\left(\mathrm{kg} / \mathrm{m}^{2}\right)$ & $26(23-28)$ & $26(24-28)$ & 0.46 \\
\hline HHS preoperatively & $59(43-67)$ & $62(48-69)$ & 0.25 \\
\hline HHS postoperatively (3.3y FU) & $97(93-100)$ & $97(92-100)$ & 0.89 \\
\hline HHS postoperatively (7.7y FU) & $98(93-100)$ & $96(91-100)$ & 0.47 \\
\hline UCLA Score preoperatively & $4(3-6)$ & $5(3-6)$ & 0.15 \\
\hline UCLA Score postoperatively (3.3y FU) & $7(6-7)$ & $7(6-8)$ & 0.35 \\
\hline UCLA Score postoperatively (7.7y FU) & $7(5-7)$ & $7(5-7)$ & 0.79 \\
\hline \multicolumn{4}{|l|}{ Diagnosis } \\
\hline Primary osteoarthritis & 61 & 46 & 0.71 \\
\hline Developmental dysplasia & 22 & 28 & 0.05 \\
\hline Avascular necrosis & 10 & 4 & 0.22 \\
\hline Posttraumatic osteoarthritis & 4 & 1 & 0.27 \\
\hline Rheumatoid arthritis & 5 & 4 & 0.93 \\
\hline Others & 3 & 0 & 0.12 \\
\hline
\end{tabular}

$\mathrm{CH}$ cortical hypertrophy, FU follow up; median values (interquartile range)

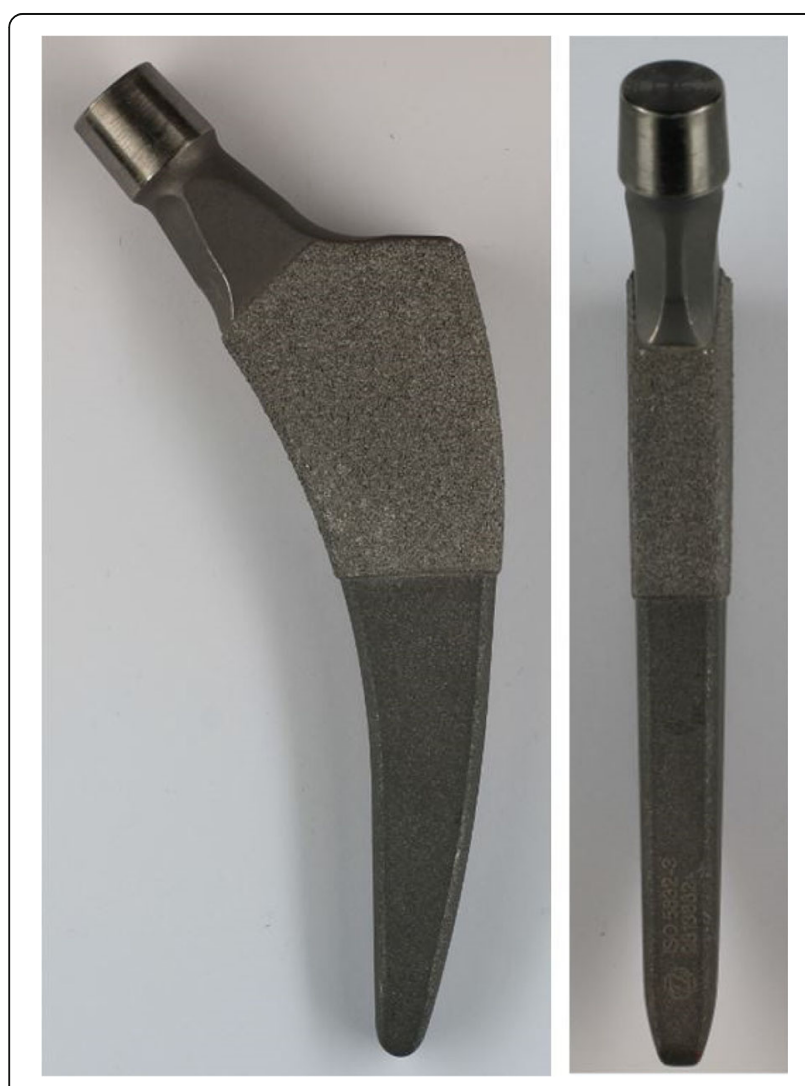

Fig. 1 Photograph of the Fitmore ${ }^{\circledast}$ hip stem bearings were used in two THAs (Biolox, CeramTec, Plochingen, Germany).

Surgery was performed by five consultant surgeons in a university hospital setting using a modified, anterolateral Watson-Jones or a transgluteal Bauer approach. Intraoperative fluoroscopy was used in all patients with the final cup implant and the templated femoral broach in situ. Surgeons aimed for secure press-fit fixation, neutral stem alignment for varus/valgus position, anteversion of the stem of $10^{\circ} \pm 10^{\circ}$, combined cup inclination/ anteversion between $40 \pm 10^{\circ} / 20 \pm 10^{\circ}$, balanced leg length and reconstruction of the hip offset [14]. Standardized preoperative planning of the prosthesis size and position was performed in all patients.

\section{Clinical and radiographic assessment}

Patients had clinical and radiographic follow-up examinations in regular intervals at 1,3 , and 5 to 10 years postoperatively. Preoperatively and at each time of follow-up, the Harris hip score (HHS) and the University of California, Los Angeles (UCLA) activity score were assessed $[15,16]$. Standardized digital, calibrated AP hip, lateral hip and low-centred AP radiographs of the pelvis were acquired [17]. Radiographs were assessed as previously described for implant loosening, radiolucent lines, osteolysis, heterotopic ossification and cortical hypertrophies by two reviewers (MMI; JW), who were not involved in index surgery and blinded to each other [5]. Implant loosening was defined by the criteria of Engh et 
al. [18]. Osteolysis was defined as area with reduced bone stock or endostal resorption. Osteolysis, radiolucent lines and cortical hypertrophies were evaluated using the zones described by Gruen et al. [19]. Heterotopic ossifications were evaluated by the criteria of Brooker et al. [20]. The methods for radiographic measurements have been described previously in detail [10]. Briefly, varus/valgus stem alignment was measured as the difference in degrees between stem axis and proximal femoral shaft axis. The Canal Fill Index (CFI) was determined, to evaluate the meta-/ diaphyseal filling of the femoral canal by the cementless stem three centimeter below the lesser trochanter $[21,22]$. Acetabular and femoral offset (AO, FO) were measured as the distance between the center of rotation of the femoral head and ipsilateral teardrop figure and the center of rotation and proximal femoral shaft axis, respectively. The postoperative offset change $(\triangle \mathrm{AO}$ and $\triangle \mathrm{FO}$ ) was determined as the difference between pre- and postoperative $\mathrm{AO}$ and FO. Hip offset was defined as the sum of AO and FO [14]. Roman software V1.70 (Institute of Orthopedics, Oswestry, UK) and ImageJ software V1.44 (National Institute of Health, USA) were used for radiographic analysis.

At last follow-up, one patient of the study cohort was lost, 14 patients (14 hips, 6\%) had died with the stem in situ, 2 patients (2 hips, 1\%) had withdrawn their study consent and 2 patients ( 2 hips, $1 \%$ ) had needed stem revision. Therefore, 214 patients (227 hips, 92\%) were available for clinical follow-up after a mean of 7.7 years (6-10 years) with 188 patients having additional up to date radiographs (Fig. 2).

\section{Statistical analysis}

After exploratory data analysis including Q-Q plots and Kolmogorov-Smirnov tests, non- parametric tests were used. Values are given as medians with interquartile range. To answer the first study question, group comparisons were performed using the Wilcoxon log-rankor the Mann-Whitney-U-Test and stem survival was estimated using Kaplan-Meier survival analysis with 95\% confidence intervals (CI). Patients were censored at death, at stem revision for different endpoints or at the end of follow-up, whichever came first. With regard to sample size, the survival was calculated to 8.6 years with a minimum of 17 hips still being followed up [23]. To answer the second study question, 171 hips could be included to calculate the odds ratios for potential risk factors being associated with the occurrence of a $\mathrm{CH}$ using a logistic regression model (in 11 patients with bilateral THAs, only the first hip was included and 6 patients had incomplete data sets, leaving 171 patients for analysis). A sample size calculation was performed according to the formula by Peduzzi et al. $(n=$ $10 \times \mathrm{k} \div \mathrm{p}$ ) [24]. Based on the percentage (p) of $63 \%$ for $\mathrm{CHs}$ found in a previous study [5], and six predictors (k) being included in the regression model, a minimum of 95 hips would be needed to achieve sufficient power. We considered $p$-values of $<0.05$ to be significant. SPSS $^{\circ}$ Version 24.0 (IBM SPSS Statistics, IBM, Armonk, NY, USA) and GraphPad Prism ${ }^{\circ}$ Version 6.0 (GraphPad Software, San Diego, CA) were used to record and analyze the collected data. Intra- and interobserver reliabilities were calculated for 24 randomly selected data sets $(10 \%$ of THAs), using averagemeasureintraclass-correlation coefficients (ICC) with a two-way random effects model for absolute agreement. Repeated measurements for intra- observer reliability were performed at day 1 and day 7 in a blinded fashion. The inter- and intra-observer correlation coefficients were excellent for radiographic measurements (range, $0.931 \quad(95 \% \mathrm{CI} ; 0.813-0.972)$ to 0.997 (95\% CI; 0.994-0.999).

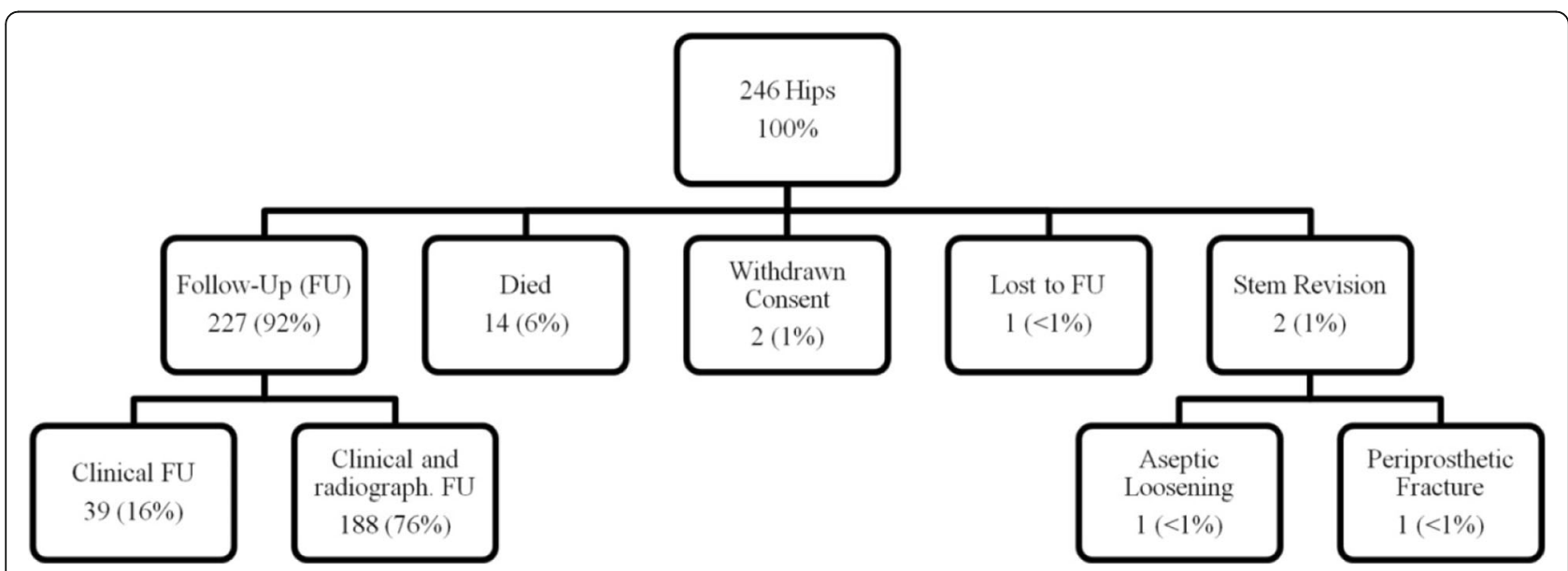

Fig. 2 Distribution of hips at last Follow-Up (FU). Clinical follow up included data on patient reported outcome measures, thigh pain and stem survival. In patients with clinical and radiographic FU, additional up to date radiographs were available 


\section{Results}

Cortical hypertrophies were observed in $56 \%$ of the hips $(n=105)$ and mostly distally located in Gruen zone 3 and 5, indicating a slight increase in the percentage of $\mathrm{CHs}$ compared to previous follow-up (53\% after 3.3 years). The distribution and change of radiolucencies and hypertrophies are shown in Fig. 3. There was no significant difference for the HHS and UCLA score for patients with and without CHs (HHS: 98 (93-100) vs. 96 (91-100) points, $p=0.47$ and UCLA: $7(5-7)$ vs. 7 (5-7), $(p=0.79$; (median (IQR)). One patient reported thigh pain. In this patient a mild $\mathrm{CH}$ was observed in Gruen zone 5 but extensive heterotopic ossification around the neck of the stem. No patient with a decrease in hip offset by more than $1 \mathrm{~cm}$ showed a limp and no patient with an increase in offset by more than $5 \mathrm{~mm}$ showed symptoms of a trochanteric bursitis. One stem revision was performed after 1.8 years for aseptic loosening due to continuous subsidence without primary fixation. A second stem revision was performed after a fall on the hip after 8.6 years for a periprosthetic Vancouver B2 fracture. The Kaplan Meier survival rate after 8.6 years was $99.6 \%$ (95\%-CI; 97.1-99.9\%) for the endpoint "stem revision due to aseptic loosening" and 93.7\% (95\%-CI, 66.5-98.9\%) for the endpoint "all stem revisions" and no association with $\mathrm{CHs}$ and stem revision could be detected (Fig. 4 a\&b).
An increase in hip offset was identified as the only risk factor for the development of $\mathrm{CHs}$ in the regression model $(\Delta \mathrm{HO}$; OR $1.1(1.0-1.2) ; p=0.001)$ (Table 2$)$. Regarding effect size, median $\Delta \mathrm{HO}$ was $-0.3 \mathrm{~mm}(-7.7-$ -3.8) for hips with and $-3.8 \mathrm{~mm}(-4.9-3.5)$ without $\mathrm{CHs}$. The proportion of hips with $\mathrm{CHs}$ steadily increased with the increase in hip offset and only the two subgroups with "under-reconstructed" hip offset $(\Delta \mathrm{HO}<-$ $2.5 \mathrm{~mm}$ ) showed a proportion of $<50 \%$ for hips with CHs (Figs. 5 and 6).

\section{Discussion}

The concept of short femoral stems was recently described as being attractive, but concerns were expressed due to a lack of data in the literature and more structured and specific research was recommended [25]. It was hypothesized that in the future, short stems may become the norm, but we were a long way from that [25]. In the face of this current trend, the present findings regarding clinical outcome, thigh pain, stem survival and cortical hypertrophies are of clinical relevance.

To our knowledge, this is the first study reporting a constant high percentage of $56 \%$ of cortical hypertrophies for this specific cementless curved, short stem design in the mid-term after 6 to 10 years compared to the short term after 2 to 4 years. Despite the present radiographic findings, there was no difference in clinical

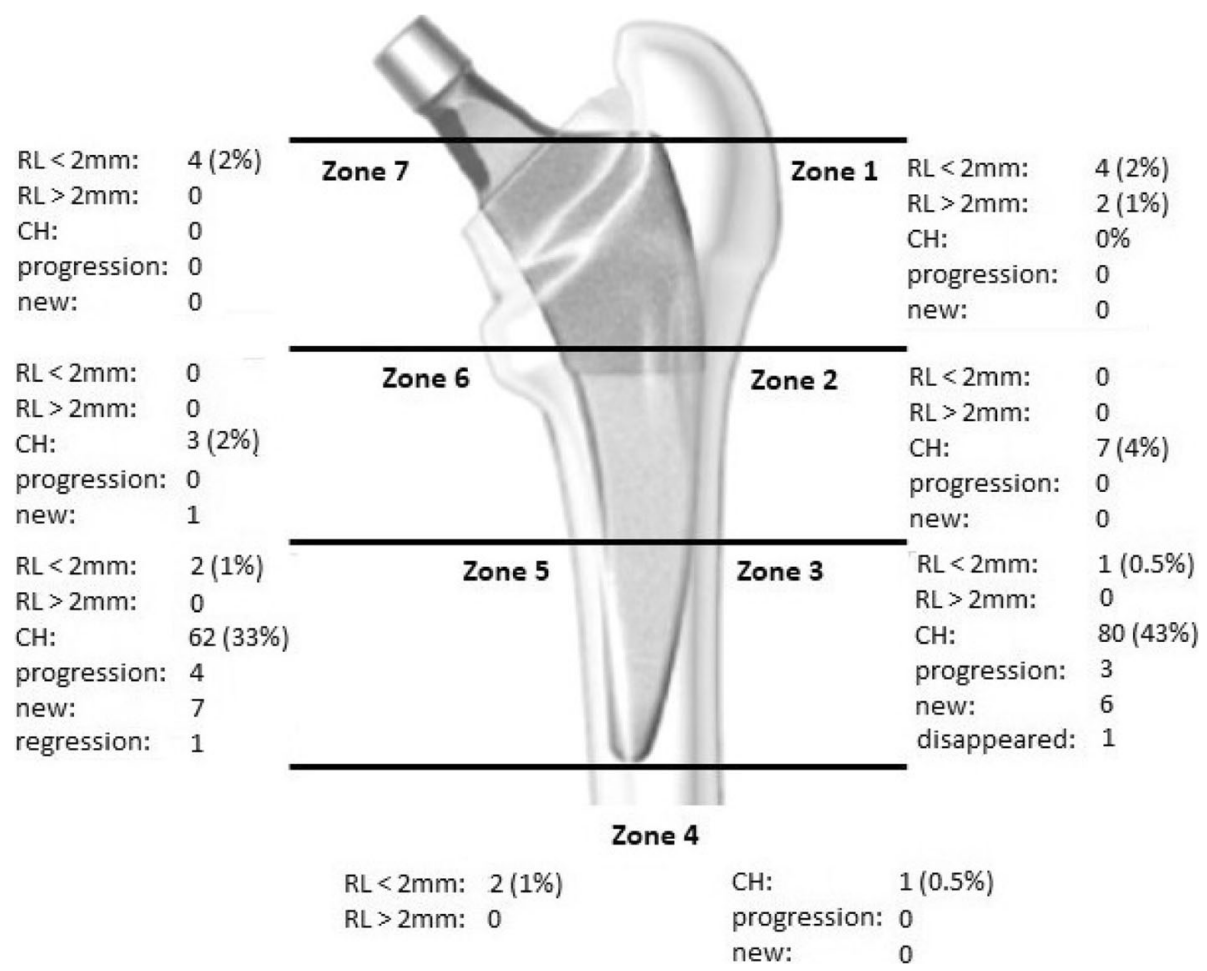

Fig. 3 Distribution of radiolucencies (RL) and cortical hypertrophies $(\mathrm{CH})$ around the 188 hip stems with available radiographic follow-up. Figure adapted according to Maier et al., 2015, BMC Musculoskeletal Disorders [5] 

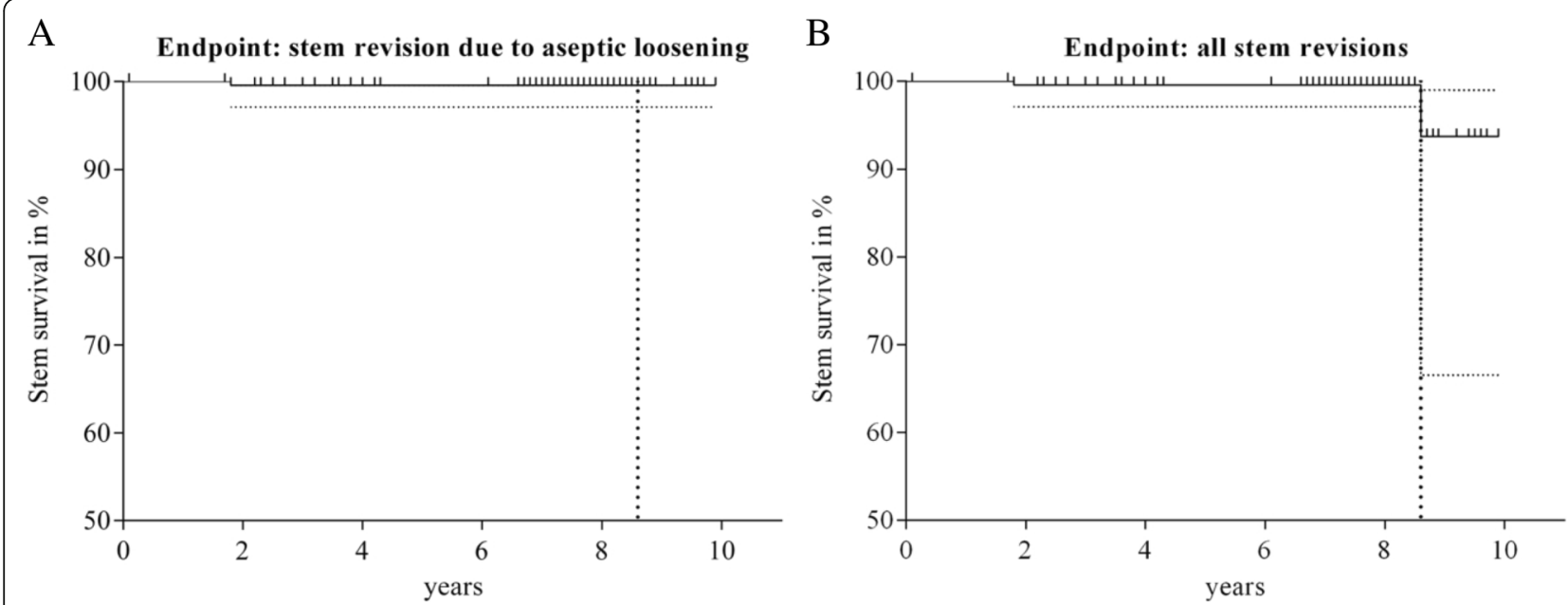

Fig. 4 a\&b: Kaplan Meier survival rate after 8.6 years for the endpoint A: "stem revision due to aseptic loosening (99.6\%; 95\%-Cl; 97.1-99.9\%) and for B: "all stem revisions" (93.7\%; 95\%-Cl; 66.5-98.9\%) $(n=246)$

outcome and stem survival for patients with and without $\mathrm{CHs}$ and the only patient or surgery-related factor being associated with the development of $\mathrm{CHs}$ was reconstruction of hip offset. Surgeons aimed to reconstruct hip offset in order to achieve optimal clinical outcome, which has been recently demonstrated to correlate with accurate $\mathrm{HO}$ reconstruction and minimized leg-length difference [14]. Under-reconstruction of hip offset should be avoided as it is associated with inferior clinical outcome and increased risk for dislocation, as soft tissue tension is reduced, while potential negative effects of a slight increase in hip offset are still controversially discussed [14, 26]. In the present study, subgroups with adequately restored to increased hip offset $(-2.5 \mathrm{~mm}$ to $>+10 \mathrm{~mm})$ demonstrated percentages of more than $50 \%$ for hips with CHs. In order to achieve optimal outcome with this short stem and reconstructing hip offset, the increased likelihood of developing $\mathrm{CHs}$ might have to be accepted. As no relevant number of radiolucencies or osteolysis could be observed in the proximal Gruen zones and stem survival was excellent in the mid-term, we suggest interpreting the high percentage of $\mathrm{CHs}$ as bone

Table 2 Logistic regression model expressing the increased likelihood for the development of $\mathrm{CH}$ s dependent on hip offset reconstruction. (Nagelkerkes $\mathrm{R}^{2}=0.118$ )

\begin{tabular}{lll}
\hline Model $(n=171)$ & Odds Ratio $(95 \%-\mathrm{Cl})$ & $p$-value \\
\hline Stem axis & $1.007(0.881-1.151)$ & 0.923 \\
BMI & $0.988(0.921-1.059)$ & 0.733 \\
Age at surgery in years & $0.989(0.962-1.016)$ & 0.405 \\
Gender & $0.608(0.306-1.21)$ & 0.157 \\
Canal Fill Index & $1.817(0.092-36.007)$ & 0.581 \\
$\Delta$ Hip Offset in mm & $1.104(1.044-1.168)$ & $0.001^{*}$ \\
\hline
\end{tabular}

*indicating significance $(p<0.05)$ remodeling due to a potential distal load transfer but not as a sign of proximal stem loosening.

Interpreting our results in context of the literature, the large variety of short hip stems and different classifications have to be acknowledged [12, 13]. Applying the classifications from the literature, the Fitmore stem can be characterized as a trochanter-sparing or neckharming cementless stem $[12,13]$. Due to the variety of "short"-stem designs with individual patterns of cementless fixation and load transfer, the comparison of different stem designs regarding bone remodeling and occurrence of cortical hypertrophies is difficult. A systematic review and meta-analysis by Yan et al. reported similar periprosthetic bone remodeling around several short- and standard stems, with moderate quality evidence [27]. In particular, bone remodeling of the Fitmore stem compared to the CLS stem demonstrated significantly less bone mineral density reduction in the distal, lateral femur, equivalent to Gruen zones $2 \& 3$ in a prospective randomized study [28]. This finding supports our assumption, that the present distal femoral cortical hypertrophies might be attributable to bone remodeling with pronounced distal load transfer. However, surgeons should be aware that asymptomatic $\mathrm{CHs}$ should be distinguished from symptomatic $\mathrm{CHs}$ presenting with thigh pain because stress fractures around a short cementless stem without trauma have been reported in rare cases [29]. Stem survival was good in the present study with 93.7\% after 8.6 years for the endpoint all stem revisions comparing well to other trochanter sparing stem designs with survival rates of 90 to $98 \%$ after 7 years [12]. Furthermore, no trend towards an increased revision rate for stems with $\mathrm{CHs}$ could be observed in our study while the rate of $\mathrm{CHs}$ remained almost constant at $56 \%$ after 7.7 years compared to $53 \%$ after 3.3 years. With respect 


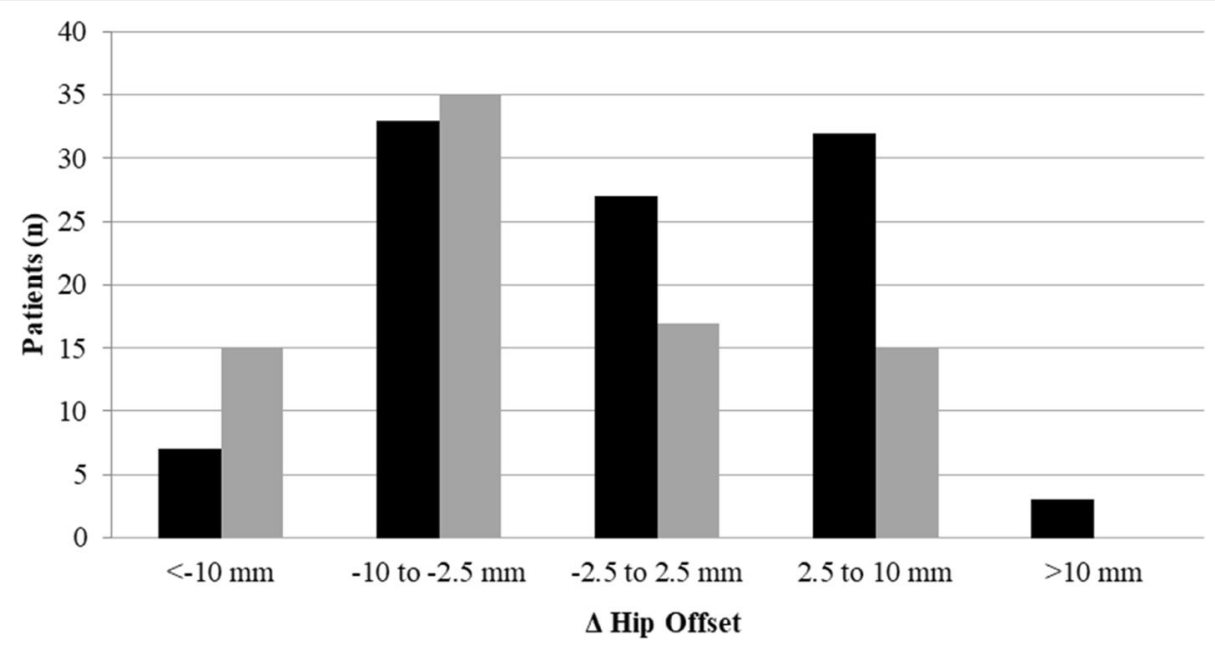

- Hips with $\mathrm{CH} \quad$ Hips without $\mathrm{CH}$

Fig. 5 Histogram showing the distribution and proportion of hips with and without $\mathrm{CH}$ s depending on hip offset reconstruction ( $\Delta \mathrm{HO})$. Patients with adequate or over-reconstructed hip offset demonstrated a higher proportion of hips with cortical hypertrophies $(n=188)$

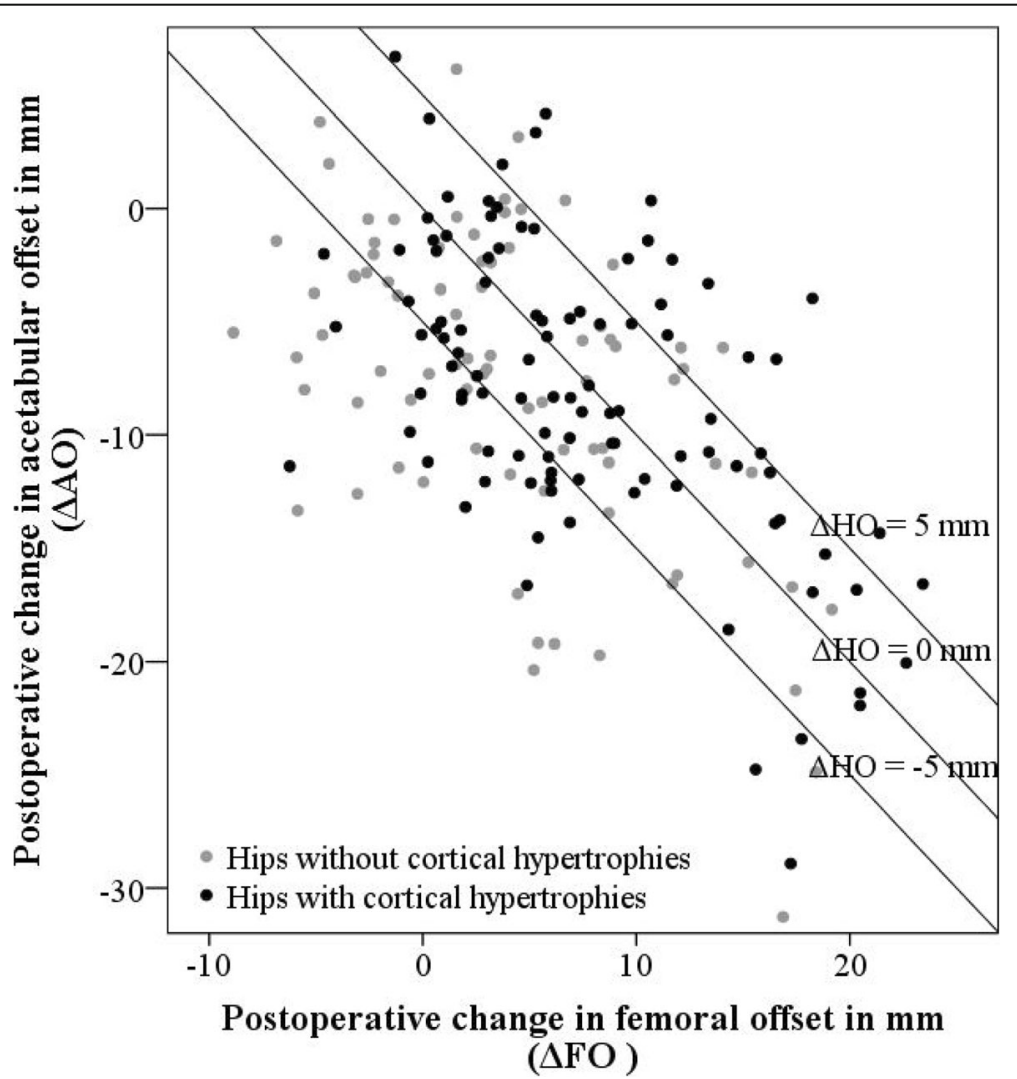

Fig. 6 Scatter plot showing hips with (black) and without $\mathrm{CH}$ (grey), dependent on change in hip offset reconstruction, with hip offset being the sum of femoral and acetabular offset $(\Delta \mathrm{HO}=\Delta \mathrm{FO}+\Delta \mathrm{AO})(n=188)$ 
to clinical outcome, no difference could be demonstrated for the HHS and UCLA score for patients with and without $\mathrm{CHs}$ in the mid-term while thigh pain was observed in only one patient.

The study has limitations that have to be addressed. During the study period 246 of 836 THAs were performed with the studied short stem and 506 with a cementless tapered straight stem. The reviewed hip stem was chosen by surgeons over the standard tapered straight stem, when offering a more precise option of press-fit fixation and hip anatomy reconstruction during templating. Therefore, a potential preoperative selection bias cannot be excluded due to the retrospective study design, but we tried to minimize this bias by including all patients with the short stem during the study period. A further limitation due to the retrospective study design is that no bone mineral density measurements were performed preoperatively and consecutively thereafter. Nevertheless, the percentage of $76 \%$ for clinical and radiological follow-up was high and only one patient $(0.5 \%)$ lost. In combination with the excellent ICCs, indicating high reliability of the radiographic measurements, the present study was adequately designed and powered to answer our study questions. Although, the regression analysis demonstrated that hip offset restoration was the only risk factor being associated with the development of $\mathrm{CHs}$, the explanatory power of the model and clinical relevance of hip offset restoration for the occurrence of cortical hypertrohies has to be interpreted with caution due to the small $\mathrm{R}^{2}$-value $(0.118)$ and the wide $95 \%$-CI for the canal fill index. Therefore, the model might be underpowered for the factor canal fill index. Lastly, stem design results in an individual fixation pattern and load transmission to the proximal femur. Therefore, care must be taken when applying the presented findings on cortical hypertrophies and thigh pain to different stem designs.

\section{Conclusion}

Excellent clinical outcome, without differences for thigh pain and stem survival was observed for patients with and without $\mathrm{CHs}$. The only patient and surgery-related factor being associated with the development of $\mathrm{CHs}$ was the change in hip offset. Therefore, we assume that the high percentage of cortical hypertrophies is not a cause for concern with this specific stem design in the midterm, but long term follow-up is needed to confirm this assumption.

\section{Abbreviations}

AO: Acetabular offset; CFI: Canal Fill Index; CH: Cortical hypertrophy; Cl: Confidence intervals; Fig.: Figure; FO: Femoral offset; HHS: Harris Hip Score; HO: Hip offset; ICC: Intraclass-correlation coefficients; IQR: Interquartile range; OR: Odds ratio; THA: Total hip arthroplasty; UCLA: University of California, Los Angeles: $\triangle \mathrm{AO}$ : Postoperative change in acetabular offset compared to preoperatively; $\Delta \mathrm{FO}$ : Postoperative change in femoral offset compared to preoperatively; $\Delta \mathrm{HO}$ : Postoperative change in hip offset compared to preoperatively

\begin{abstract}
Acknowledgements
We thank Ms. Marlies Krueger for data acquisition from the institutional database, our clinical study assistant Ms. Emanuela Dabbeni helping to conduct the study and Ms. Ulrike Mueller from the Laboratory of Biomechanics and Implant Research at our department for taking the photographic image of the hip stem.

We acknowledge financial support by Deutsche Forschungsgemeinschaft within the funding programme Open Access Publishing, by the BadenWürttemberg Ministry of Science, Research and the Arts and by RuprechtKarls-Universität Heidelberg.
\end{abstract}

\section{Authors' contributions}

MMI: data acquisition, analysis and interpretation of data, drafting and critical revision of the manuscript. JW: data acquisition, measurements, data analysis, critical revision of the manuscript. TB: Statistical consultation, design and interpretation of data, critical revision of the manuscript. MRS: data acquisition, and interpretation of data, critical revision of the manuscript. TW: data acquisition, and interpretation of data, critical revision of the manuscript. TG: data acquisition, and interpretation of data, critical revision of the manuscript. CM: design of the study and interpretation of data, critical revision of the manuscript. MM: design, data acquisition, analysis and interpretation of data, drafting and critical revision of the manuscript. All authors read and approved the final manuscript.

\section{Funding}

We would like to thank the non-commercial research fund Deutsche Arthrose-Hilfe e.V. for supporting this study. The funding body had no influence on the design of the study, collection, analysis, interpretation of data and writing the manuscript.

\section{Availability of data and materials}

The dataset used and analysed in the current study is available from the corresponding author on reasonable request.

\section{Ethics approval and consent to participate}

Written informed consent was obtained by all patients. The study was submitted to and approved by the ethics board of the University of Heidelberg (S-083/2017), including the methods and ethics of the study. The study conducted according to the Helsinki Declaration of 2008.

\section{Consent for publication}

Not applicable.

\section{Competing interests}

CM and TG report grants paid to their institution during the conduct of the study from Zimmer Biomet, Europe and from Depuy Synthes Orthopädie Gmbh, outside the submitted work and receiving personal speaker fees and institutional support during the conduct of the study from Zimmer Biomet and AE Germany outside the submitted work. The authors declare that they have no competing interests.

\section{Author details}

${ }^{1}$ Department of Orthopaedics and Trauma Surgery, Heidelberg University Hospital, Schlierbacher Landstrasse 200a, 69118 Heidelberg, Germany. ${ }^{2}$ Institute of Medical Biometry and Informatics, University of Heidelberg, Im Neuenheimer Feld 130.3, 69120 Heidelberg, Germany. ${ }^{3}$ Department of Orthopaedic and Trauma Surgery, Kepler University Hospital, Med Campus III Krankenhausstraße 9, 4021 Linz, Austria.

Received: 19 December 2018 Accepted: 20 May 2019

Published online: 29 May 2019

\section{References}

1. Hutt J, Harb Z, Gill I, Kashif F, Miller J, Dodd M. Ten year results of the collum femoris preserving total hip replacement: a prospective cohort study of seventy five patients. Int Orthop. 2014;38(5):917-22. 
2. Kendoff DO, Citak M, Egidy CC, O'Loughlin PF, Gehrke T. Eleven-year results of the anatomic coated CFP stem in primary total hip arthroplasty. Arthroplast. 2013;28(6):1047-51.

3. Nowak M, Nowak TE, Schmidt R, Forst R, Kress AM, Mueller LA. Prospective study of a cementless total hip arthroplasty with a collum femoris preserving stem and a trabeculae oriented pressfit cup: minimun 6-year follow-up. Arch Orthop Trauma Surg. 2011;131(4):549-55.

4. Briem D, Schneider M, Bogner N, Botha N, Gebauer M, Gehrke T, Schwantes B. Mid-term results of 155 patients treated with a collum femoris preserving (CFP) short stem prosthesis. Int Orthop. 2011;35(5):655-60.

5. Maier MW, Streit MR, Innmann MM, Kruger M, Nadorf J, Kretzer JP, Ewerbeck $\checkmark$, Gotterbarm T. Cortical hypertrophy with a short, curved uncemented hip stem does not have any clinical impact during early follow-up. BMC Musculoskelet Disord. 2015;16(1):371.

6. Giardina F, Castagnini F, Stea S, Bordini B, Montalti M, Toni A. Short stems versus conventional stems in Cementless Total hip arthroplasty: a long-term registry study. J Arthroplast. 2018;33(6):1794-9.

7. Amendola RL, Goetz DD, Liu SS, Callaghan JJ. Two- to 4-year Followup of a short stem THA construct: excellent fixation, thigh pain a concern. Clin Orthop Relat Res. 2017;475(2):375-83.

8. Gustke K. Short stems for total hip arthroplasty: initial experience with the Fitmore stem. J Bone Joint Surg Br. 2012;94(11 Suppl A):47-51.

9. Pepke W, Nadorf J, Ewerbeck V, Streit MR, Kinkel S, Gotterbarm T, Maier MW Kretzer JP. Primary stability of the Fitmore(R) stem: biomechanical comparison. Int Orthop. 2014;38(3):483-8.

10. Innmann MM, Spier K, Streit MR, Aldinger PR, Bruckner T, Gotterbarm T, Merle C. Comparative analysis of the reconstruction of individual hip anatomy using 3 different Cementless stem designs in patients with primary hip osteoarthritis. J Arthroplast. 2018;33(4):1126-32.

11. Gasbarra E, Celi M, Perrone FL, lundusi R, Di Primio L, Guglielmi G, Tarantino U. Osseointegration of Fitmore stem in total hip arthroplasty. J Clin Densitom. 2014;17(2):307-13.

12. van Oldenrijk J, Molleman J, Klaver M, Poolman RW, Haverkamp D. Revision rate after short-stem total hip arthroplasty: a systematic review of 49 studies. Acta Orthop. 2014;85(3):250-8.

13. Hauer G, Vielgut I, Amerstorfer F, Maurer-Ertl W, Leithner A, Sadoghi P. Survival rate of short-stem hip prostheses: a comparative analysis of clinical studies and National Arthroplasty Registers. J Arthroplast. 2018;33(6):1800-5.

14. Innmann MM, Maier MW, Streit MR, Grammatopoulos G, Bruckner T, Gotterbarm T, Merle C. Additive influence of hip offset and leg length reconstruction on postoperative improvement in clinical outcome after Total hip arthroplasty. J Arthroplast. 2018;33(1):156-61.

15. Harris WH. Traumatic arthritis of the hip after dislocation and acetabular fractures: treatment by mold arthroplasty. An end-result study using a new method of result evaluation. J Bone Joint Surg Am. 1969;51(4):737-55.

16. Zahiri CA, Schmalzried TP, Szuszczewicz ES, Amstutz HC. Assessing activity in joint replacement patients. J Arthroplast. 1998;13(8):890-5.

17. Dastane M, Dorr LD, Tarwala R, Wan Z. Hip offset in total hip arthroplasty: quantitative measurement with navigation. Clin Orthop Relat Res. 2011;469(2):429-36.

18. Engh CA, Bobyn JD, Glassman AH. Porous-coated hip replacement. The factors governing bone ingrowth, stress shielding, and clinical results. J Bone Joint Surg Br. 1987;69(1):45-55.

19. Gruen TA, McNeice GM, Amstutz HC. "modes of failure" of cemented stemtype femoral components: a radiographic analysis of loosening. Clin Orthop Relat Res. 1979;141:17-27.

20. Brooker AF, Bowerman JW, Robinson RA, Riley LH Jr. Ectopic ossification following total hip replacement. Incidence and a method of classification. J Bone Joint Surg Am. 1973;55(8):1629-32.

21. Streit MR, Innmann MM, Merle C, Bruckner T, Aldinger PR, Gotterbarm T. Long-term (20- to 25-year) results of an uncemented tapered titanium femoral component and factors affecting survivorship. Clin Orthop Relat Res. 2013:471(10):3262-9

22. Laine HJ, Pajamaki KJ, Moilanen T, Lehto MU. The femoral canal fill of two different cementless stem designs. The accuracy of radiographs compared to computed tomographic scanning. Int Orthop. 2001;25(4):209-13.

23. Dorey FJ. Survivorship analysis of surgical treatment of the hip in young patients. Clin Orthop Relat Res. 2004;418:23-8.

24. Peduzzi P, Concato J, Feinstein AR, Holford TR. Importance of events per independent variable in proportional hazards regression analysis. II. Accuracy and precision of regression estimates. J Clin Epidemiol. 1995;48(12):1503-10.
25. Pairon P. Stem size in hip arthroplasty. Bone Joint J. 2018;100-B(9):1133-5.

26. Robinson M, Bornstein L, Mennear B, Bostrom M, Nestor B, Padgett D, Westrich $G$. Effect of restoration of combined offset on stability of large head THA. Hip Int. 2012;22(3):248-53.

27. Yan SG, Li D, Yin S, Hua X, Tang J, Schmidutz F. Periprosthetic bone remodeling of short cementless femoral stems in primary total hip arthroplasty: a systematic review and meta-analysis of randomizedcontrolled trials. Medicine (Baltimore). 2017;96(47):e8806.

28. Freitag $T$, Hein MA, Wernerus D, Reichel H, Bieger R. Bone remodelling after femoral short stem implantation in total hip arthroplasty: 1-year results from a randomized DEXA study. Arch Orthop Trauma Surg. 2016;136(1):125-30.

29. Slullitel PA, Onativia Jl, Llano L, Comba F, Zanotti G, Piccaluga F, Buttaro MA Periprosthetic stress fracture around a well-fixed type $2 \mathrm{~B}$ short uncemented stem. SICOT J. 2018;4:33.

\section{Publisher's Note}

Springer Nature remains neutral with regard to jurisdictional claims in published maps and institutional affiliations.
Ready to submit your research? Choose BMC and benefit from:

- fast, convenient online submission

- thorough peer review by experienced researchers in your field

- rapid publication on acceptance

- support for research data, including large and complex data types

- gold Open Access which fosters wider collaboration and increased citations

- maximum visibility for your research: over $100 \mathrm{M}$ website views per year

At BMC, research is always in progress.

Learn more biomedcentral.com/submissions 